\title{
Angiotensin II-induced changes in cochlear blood flow and blood pressure in normotensive and spontaneously hypertensive rats
}

\author{
Wayne S. Quirk ${ }^{1}$, John W. Wright ${ }^{1}$, Harold A. Dengerink ${ }^{1}$ and Josef M. Miller ${ }^{2}$ \\ ${ }^{\prime}$ Department of Psychology, Washington State University, Pullman, Washington, U.S.A. \\ and ${ }^{2}$ Kresge Hearing Research Institute, University of Michigan, Ann Arbor, Michigan, U.S.A.
}

(Received 8 July 1987; accepted 6 January 1988)

\begin{abstract}
Previous investigations in our laboratory have measured significant increases in the circulating levels of the potent vasoconstrictive hormone, angiotensin II (AII; 26 and $64 \mathrm{pg} / 100 \mu 1$ plasma, normal and noise exposed, respectively), during and following noise exposure in the alert rat (Wright et al., 1981). In the present study, these levels were approximated through intra-arterial infusion in the anesthetized spontaneously hypertensive rat (SHR) and normotensive Wistar-Kyoto (WKY) rat. Laser Doppler flowmeter measurements of cochlear blood flow (CBF) indicated that despite equivalent AII-induced elevations in systemic blood pressure, CBF in the SHR did not increase to the levels measured in the WKY. Pretreatment with the specific angiotensin receptor antagonist sarile, ( Sar $^{1}$,Ile ${ }^{8}$-AII), reduced AII-induced elevations in systemic blood pressure in members of both strains, but did not change the overall pattern of CBF. These results indicate that SHRs may have a compromised cochlear circulation that is refractory to increases in systemic blood pressure.
\end{abstract}

Cochlear blood flow; Blood pressure; Laser Doppler flowmeter; Spontaneously hypertensive rat; Angiotensin

\section{Introduction}

Certain otopathological conditions, such as Meniere's disease (Williams, 1965) and noise-induced hearing loss (Borg, 1981) have been proposed to be the result of altered blood flow through the cochlear microvasculature. The decrease in oxygen and nutrients transported to the cochlea, and waste products removed from this organ, may result in hair cell fatigue and damage (Borg, 1981). The laser Doppler flowmeter is a promising technique currently being utilized to measure cochlear blood flow (CBF). The application of the laser Doppler flowmeter allows direct and dynamic measurement of changes in CBF and is relatively noninvasive (Goodwin et al., 1984; Miller et al., 1983; Miller et al., 1984). Previously we have

Correspondence to: John W. Wright, Department of Psychology, Washington State University, Pullman, WA 991644830, U.S.A. utilized the laser Doppler flowmeter to investigate the effects of vasoactive substances such as angiotensin (Wright et al., 1985a) and nicotine (Dengerink et al., 1985), and therapeutic agents such as mannitol (Goodwin et al., 1984; Quirk et al., submitted) on CBF. These studies utilized normotensive guinea pigs and rats and generally indicated that increases in systemic blood pressure were followed by increases in CBF. Less attention has been given to the relationship between systemic blood pressure and CBF in hypertensive animal models.

The spontaneously hypertensive rat (SHR) has been used as a model of human essential hypertension (Yamori, 1983). These animals have been shown to suffer greater hearing loss than normotensive rats following exposure to noise (Borg, 1981; Pillsbury, 1986), and they reveal histological changes consistent with decreased cochlear circulation following noise exposure (Axelsson et al., 1983). Some epidemiological studies concerned with the relationship between noise exposure and 
hypertension have reported no significant relationship (e.g. Drettner et al., 1975), while others have found a significant correlation among hypertensive factory workers (Johnsson and Hansson, 1977; Parvispoor, 1976). We presently utilized the SHR to test the hypothesis that hypertension produces a compromised CBF that is less responsive to elevations in systemic blood pressure as compared with normotensive Wistar-Kyoto (WKY) rats. It has been shown that during intense noise exposure circulating AII levels are increased in humans (Dengerink et al., 1982) and normotensive rats (Wright et al., 1981). These elevations were sufficient to increase systemic blood pressure and lead to an increased CBF when approximated by exogenous infusion independent of noise (Wright et al., 1981).

In the present study we questioned whether CBF responsiveness in SHRs is similar to that observed in normotensive animals. We chose to experimentally alter the circulating levels of angiotensin through intra-arterial infusion of angiotensin. We predicted that CBF would increase in both the SHR and normotensive animals in response to increases in systemic pressure induced by angiotensin infusion, but would not be as great in the SHR. Moreover, pretreatment with an angiotensin receptor antagonist was expected to reduce systemic pressure and CBF in members of both strains.

\section{Method}

Male SHR and WKY rats were derived from stock obtained from Taconic Farms and were maintained at $21-22^{\circ} \mathrm{C}$ in group cages under a 12:12 h light-dark cycle initiated at $07.00 \mathrm{~h}$. Food and water were available ad libitum.

\section{Angiotensin infusion}

Eight rats from each strain were anesthetized with Ketamine (Bristol-Myers, Inc.) and Rompun (Haver, Inc.) (100 and $2 \mathrm{mg} / \mathrm{ml}$ respectively; 1 $\mathrm{ml} / \mathrm{kg}$ i.m.) and prepared with a left carotid artery catheter (PE 50, Clay Adams) for blood pressure measurement and a right brachial artery catheter (PE 10) for infusion. Details of these surgical procedures have previously been described elsewhere (Wright et al., 1985b). The right bulla was exposed and opened for access to the cochlea. The laser Doppler probe was placed on the basal turn of the cochlea. Blood pressure was monitored using a Statham transducer (Model P23AC) and a Grass Instruments polygraph (Model 7B). Baselevel measurements were obtained for blood pressure and CBF. Dose-response curves were obtained for $5 \mathrm{~min}$ infusions $(50 \mu 1 / \mathrm{min})$ of 1,10 , 100 , and $1000 \mathrm{pmol} / \mathrm{kg} / \mathrm{min}$ of AII (U.S. Biochemical) in sterile $0.15 \mathrm{M} \mathrm{NaCl}$ only. The doses were counterbalanced for ascending and descending order with a minimum of $15 \mathrm{~min}$ between doses in order to recover baselevel values.

Eight additional animals from each strain were prepared as previously described and were infused with $10 \mathrm{nmol} / \mathrm{kg} / \mathrm{min}$ of the angiotensin receptor antagonist, sarile ( $\operatorname{Sar}^{1}{ }^{1}$ Ile $^{8}$-AII, U.S. Biochemical) for $5 \mathrm{~min}(50 \mu \mathrm{l} / \mathrm{min})$. Animals were allowed 5 min recovery and subsequently infused with either 100 or $1000 \mathrm{pmol} / \mathrm{kg} / \mathrm{min}$ of AII $(50 \mu \mathrm{l} / \mathrm{min})$ for $5 \mathrm{~min}$.

\section{Statistical analyses}

Changes in blood pressure and CBF were analyzed using a one way analysis of variance (ANOVA). Pearson product moment correlation coefficients were computed to determine whether there was a linear relationship between change in CBF or blood pressure, and dose of infused AII. Each set of slopes derived from the WKY and SHR groups was then compared by repeated measures ANOVA.

\section{Results}

As expected there were pre-infusion differences in anesthetized blood pressure, with the SHR group revealing significantly higher levels than the WKY animals (mean \pm SE: $119 \pm 5.8$ and $77.5 \pm$ $6.7 \mathrm{~mm} \mathrm{Hg}$, respectively; $t=4.08, d f=14, P<$ 0.01 ). During the infusion of $1,10,100$, and 1000 pmol doses of AII, mean blood pressure increases for SHRs averaged 1.4, 12.2, 38.0 and $90.0 \mathrm{~mm}$ $\mathrm{Hg}$, respectively. The blood pressure increases for WKYs averaged $8.0,28.6,29.8$, and $76.5 \mathrm{~mm} \mathrm{Hg}$ during infusion of the same doses of AII. Although SHRs evidenced greater blood pressure increases than WKYs at the higher doses, these changes were not significantly different (Fig. 1, 

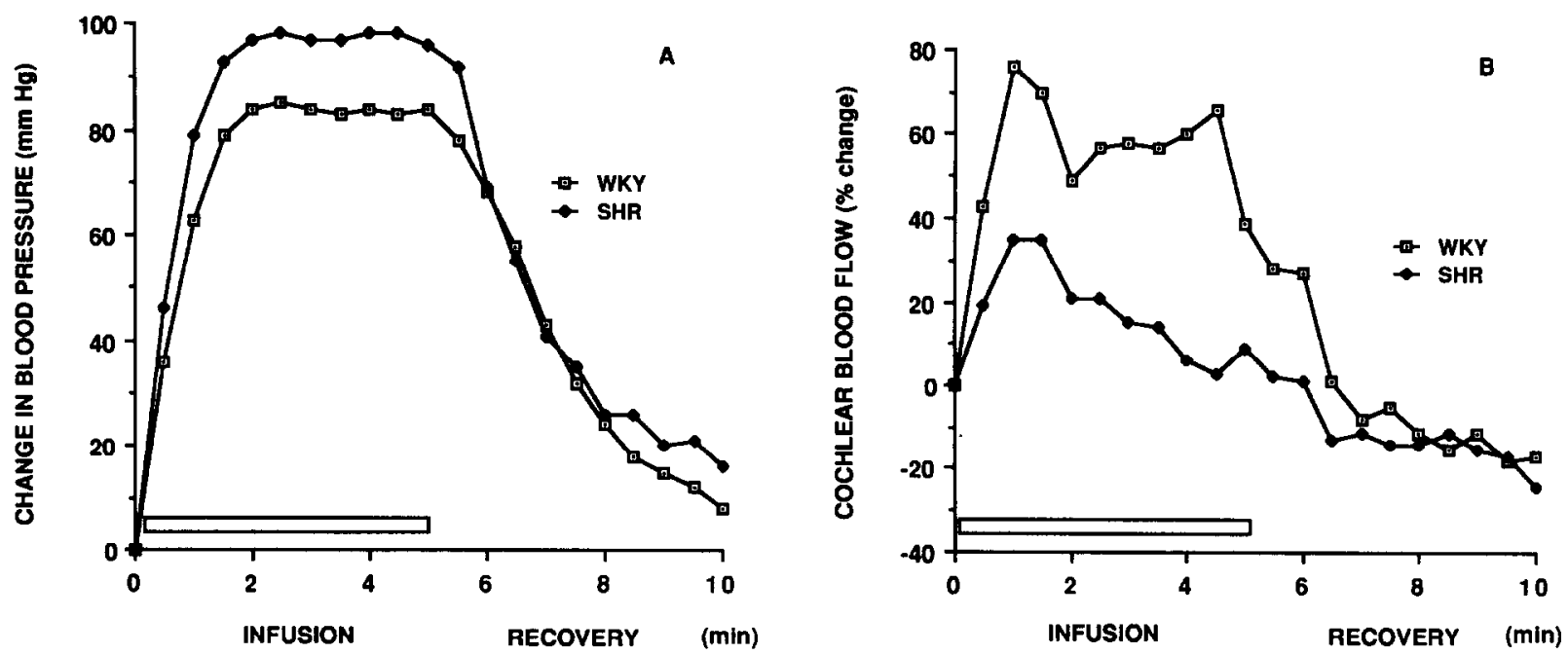

Fig. 1. Mean changes in blood pressure (Panel A) and cochlear blood flow (Panel B) induced by a 5 min infusion of AII (1000 $\mathrm{pmol} / \mathrm{kg} / \mathrm{min}$ ) in anesthetized SHR and WKY normotensive control rats (eight animals per group).

panel A). However, there were strain differences in CBF. The CBF increases for SHRs averaged $-3.1,5.7,13.6$, and $17.3 \%$ during infusion of the $1,10,100$ and $1000 \mathrm{pmol}$ doses of AII, respectively, while CBF increases observed for WKYs averaged $8.1,22.4,20.2$ and $55.5 \%$, respectively. The CBF increases observed for WKYs were significantly greater than those observed for SHRs ( $F=44.66$, $d f=1,14, P<0.01$; Fig. 1, Panel B).
Thus, WKYs revealed a consistent pattern of significant elevations in CBF concomitant with increases in blood pressure which was sustained until the last $30 \mathrm{~s}$ of the infusion. In contrast SHRs initially responded with increased CBF but then CBF slowly declined during the remainder of the infusion.

Pearson product-moment correlation coefficients were computed for blood pressure changes
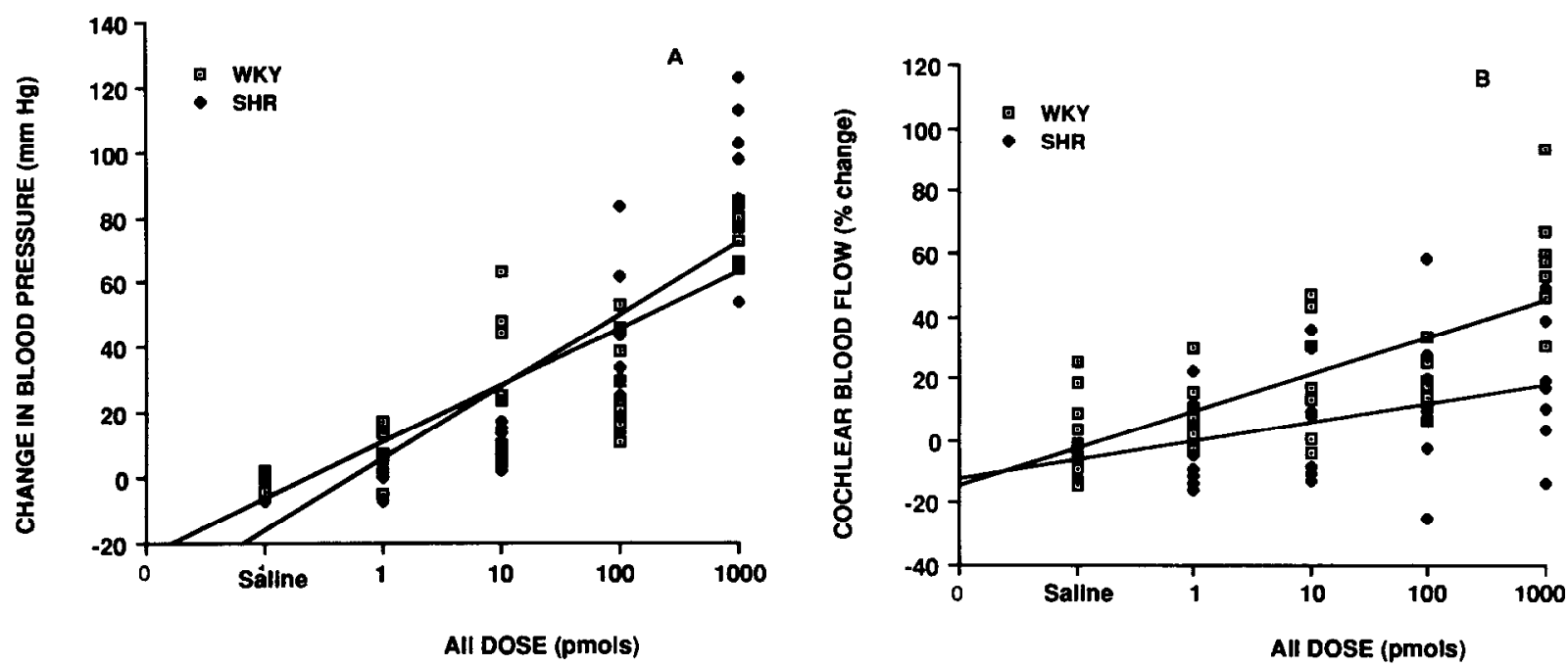

Fig. 2. Correlation of mean changes for each animal during 5 min infusion of AII (1, 10,100 and 1000 pmol) or saline for blood pressure (panel A, WKY: $y=17.46 x-23.72$; SHR: $y=21.92 x-37.67$ ) and cochlear blood flow (panel B, WKY: $y=-12.02 x-$ 14.33; SHR: $y=6.03 x-12.19)$. 
versus dose of AII (Fig. 2A) yielding coefficients of +0.92 and +0.91 for WKY and SHR groups, respectively, with each significantly different from the hypothesis that $R=0(T=5.75$ and 5.68 , respectively, $d f=38, P<0.01$ ). Analysis of variance indicated a dose-response relationship for blood pressure across groups ( $F=102.05, d f=4,56, P$ $<0.001$ ), and each dose of AII induced equivalent elevations in blood pressure in WKY and SHR groups. Thus, the pattern of AII-induced pressure elevations did not differ between the two strains. Similar Pearson product-moment correlation coefficients were computed for the data sets concerned with CBF versus dose of AII for WKY and SHR groups (Fig. 2B). The results indicated correlation coefficients of +0.85 and +0.68 for WKY and SHR groups, respectively, and each was significantly different from the hypothesis that $R=0$ ( $T=5.31$ and $T=4.25$, respectively, $d f=38, P<$ 0.01 ). Analyses of variance indicated that the WKY animals revealed significantly greater elevations in CBF than SHRs over the doses examined ( $F=$ 44.66, $d f=1,14, P<0.0001$ ).

Fig. 3 illustrates the blood pressure (panel A) and CBF (panel B) increases of SHR and WKY rats during infusion of $1000 \mathrm{pmol}$ of AII following sarile pretreatment. When pretreated with sarile the blood pressure increases during subsequent
$1000 \mathrm{pm}$ AII infusion were greater in SHRs $(\bar{X}=$ $64.7 \mathrm{~mm} \mathrm{Hg})$ than in WKY rats $(\bar{X}=49.8 \mathrm{~mm}$ $\mathrm{Hg} ; F=6.27, d f=1,14, P<0.05$ ). However, the blood pressure response to AII was reduced by approximately $30 \%$ by sarile pretreatment in both groups. The pattern of CBF change during infusion in the SHRs was an initial increase followed by a steady decline throughout the infusion period, while the WKY rat revealed sustained elevations until the infusion was terminated. Furthermore, despite sarile inhibition of AII-induced blood pressure elevations, SHRs continued to evidence smaller CBF increases (mean $=21.5 \%$ ) than did WKY rats $($ mean $=29.0 \% ; F=8.90, d f=1,14, P$ $<0.05$ ). The average increases in CBF for WKY animals were $29.0 \%$ following sarile pretreatment and $56.5 \%$ without pretreatment. In contrast, SHRs evidenced slightly greater CBF increases after sarile pretreatment $($ mean $=21.5 \%)$ than without pretreatment $($ mean $=17.3 \%)$.

\section{Discussion}

Previous investigations in our laboratory have supported the hypothesis that changes in CBF are associated with changes in systemic blood pressure (Dengerink et al., 1985; Miller et al., 1983). The data presently obtained utilizing WKY normotensive rats are consistent with this hypothesis in that
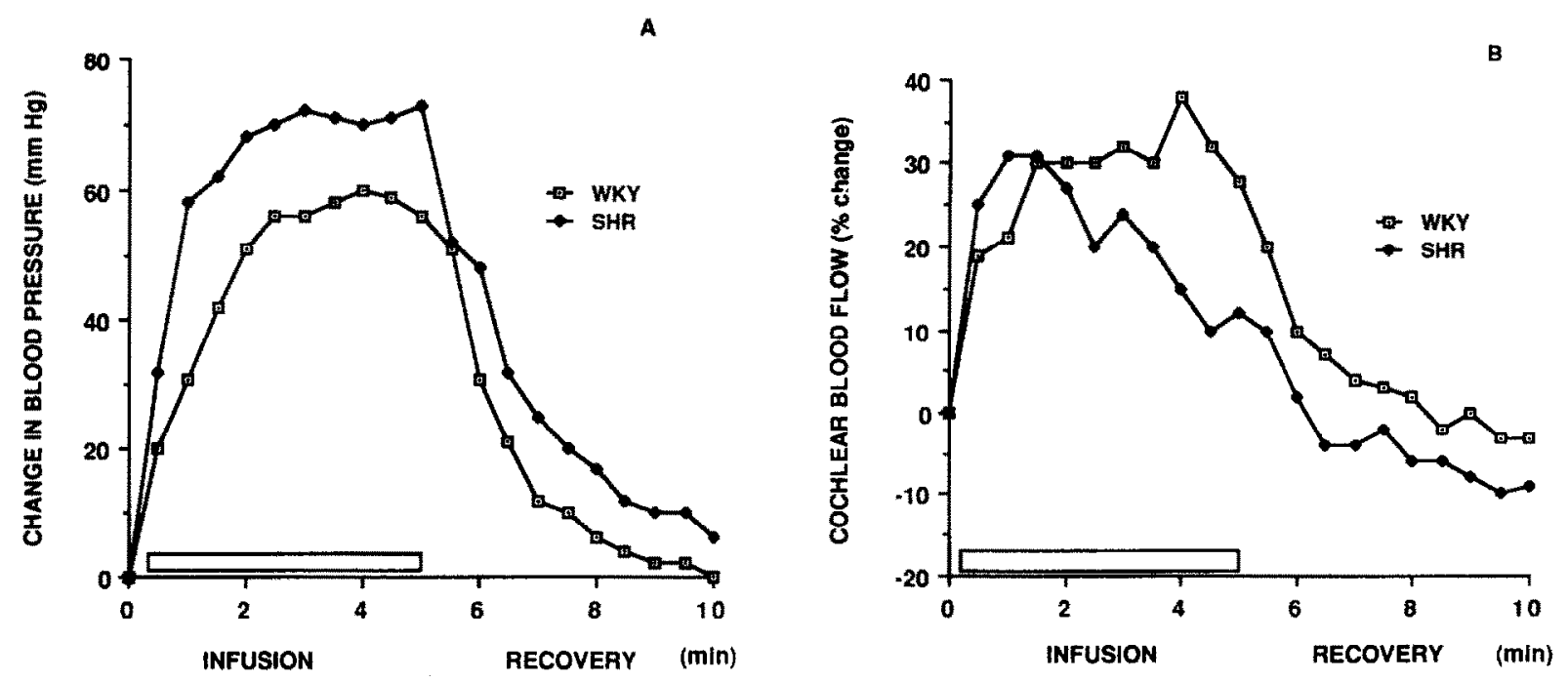

Fig. 3. Mean changes in blood pressure (Panel A) and cochlear blood flow (Panel B) induced by a 5 min infusion of AII (1000 $\mathrm{pmol} / \mathrm{kg} / \mathrm{min}$ ) following $5 \mathrm{~min}$ infusion of sarile in anesthetized SHR and WKY normotensive controls (eight animals per group). 
there was a dose dependent relationship between AII-induced increases in blood pressure and CBF. However, the results concerning blood pressure and $\mathrm{CBF}$ changes induced by AII infusion in SHRs are not consistent with previous findings for normotensive rats and guinea pigs. Specifically, despite a higher baselevel blood pressure and similar increases in blood pressure induced by AII, CBF measured in SHRs was significantly lower than CBF in WKY rats at each dose examined. Furthermore, the pattern of CBF in SHRs differed from that observed in WKY in that an initial increase was followed by a steady decline, whereas CBF in WKY increased concomitant with increases in blood pressure.

The present findings can be explained on the basis of known anatomical changes in the vasculature of the SHR and regulatory mechanisms governing blood flow in the cerebral vessels. It has been shown that adult SHRs have smaller diameter vessels and thicker vessel walls than normotensive age matched rats due to hypertrophy of the arterial walls (Harper et al., 1984). Thus, although cerebral blood flow has been reported to be within normal limits at resting blood pressure in SHRs (Barry et al., 1982), during large increases in systemic blood pressure these vessels with smaller lumen and less elastic thicker walls may not permit the same increases in flow allowed by the larger diameter vessels of normotensive animals. The expected effect due to these anatomical differences would be an initial increase followed by a plateau in CBF during sustained blood pressure elevations in the SHR. However, the present results indicate an initial peak in CBF followed by a slow persistent reduction despite sustained elevations in systemic blood pressure. These findings are more consistent with the interpretation that there may be an active mechanism that is reducing vascular perfusion of the cochlea despite persistent blood pressure elevations (reviewed by Hultcrantz and Nuttall, 1987). It is conceivable that this relative reduction of $\mathrm{CBF}$ is an adaptive response by vessels supplying blood to the cochlea. During large elevations in blood pressure this response may serve to protect the small vessels of the cochlea from large increases in perfusion pressure, thereby representing a form of vascular autoregulation.
There are several pieces of evidence from the present investigation in agreement with this vascular autoregulation interpretation. At the three highest doses of AII utilized there was an initial increase followed by a sustained reduction in CBF in SHRs. Additionally, although increases in blood pressure generally led to increases in CBF in WKYs, at the highest dose utilized there was a sharp reduction in $\mathrm{CBF}$ at $4.5 \mathrm{~min}$ of the $5 \mathrm{~min}$ infusion despite a sustained elevation in blood pressure: Therefore, an active mechanism may be evident in members of both strains with the onset of the response occurring earlier in SHRs. Furthermore, when WKY rats were pretreated with sarile, blood pressure elevations in response to AII infusions were reduced as were CBF changes. Pretreatment with sarile in SHRs also reduced AIIinduced elevations in blood pressure, but overall elevations in CBF were somewhat greater than those observed to AII infusions without sarile pretreatment. However, the pattern of reduced CBF over the infusion period was still present. Thus, sarile pretreatment inhibited AII-induced blood pressure elevations in members of both strains but had no effect on SHR CBF responses. These results are consistent with previous findings that SHRs have more vascular angiotensin II receptors than normotensive rats (Shiffrin, 1984), in that there are more potential receptor sites in the SHR than WKY animals making maximum receptor occupancy by the antagonist more likely in the WKY.

Additional evidence in favor of a vascular autoregulatory mechanism can be found in investigations of cerebral blood flow which support the existence of an autoregulatory response in cerebral vessels (Baumbach and Heistad, 1985). This mechanism is described as the adaptive reaction of blood vessels to maintain blood flow at relatively constant rates despite variations in perfusion pressure (Bayliss, 1902). Cerebral autoregulation has been demonstrated in normotensive (Coyle and Heistad, 1986) and spontaneously hypertensive rats (Baumbach and Heistad, 1985; Harper and Bohlen, 1981; Sadoshima et al., 1985), cats (Chang et al., 1985), newborn canine puppies (Pasternak and Groothuis, 1985) and baboons (Strandgaard et al., 1975). Since the cochlear vasculature is a branch of the cerebral circulation, it is not surpris- 
ing to find evidence for the presence of autoregulation in this system.

In sum, the present results identify a persistent difference in CBF between SHR and WKY rats as induced by intra-arterial infusion of AII that may be representative of a larger phenomenon involving the entire cerebral vasculature. At this time it is not clear whether an increase in CBF equivalent to the increase in systemic blood pressure, as measured in WKY rats, is a physiologically more appropriate response than the adjustment in CBF observed in the SHR. It is also important to point out that the laser Doppler probe, in its present configuration, measures changes in CBF only at the basal turn of the cochlea; thus blood flow changes in the remainder of the cochlea are not monitored. However, Nuttall (1987) has recently utilized intravital microscopy to determine that the flow velocities of fluorescently-labeled red blood cells are constant throughout the lateral wall of the cochlea. If we accept the assumption that cochlear blood flow is similar to cerebral blood flow (Lawrence et al., 1977), then it may be appropriate to suggest that the reduced CBF presently measured in the spontaneously hypertensive rat could prevent necessary perfusion of the cochlea during times of high metabolic need, such as the increases in blood pressure that accompany exposure to noise, and thus contribute to the noise-induced hearing loss previously reported in members of this strain.

\section{Acknowledgements}

This research was supported by the Deafness Research Foundation, the American Heart Association and its Washington Affiliate (831145), NIH Grant RO-HL32063 and by funds provided by Washington State University.

\section{References}

Axelsson, A., Borg, E. and Hornstrand, C. (1983) Noise effects on the cochlear vasculature in normotensive and spontaneously hypertensive rats. Acta Otolaryngol. 96, 215-225.

Barry, D., Strandgaard, S. and Graham, D. (1982) Cerebral blood flow in rats with renal and spontaneous hypertension: resetting of the lower limit of autoregulation. J. Cereb. Blood Flow Metab. 347-353.

Baumbach, G. and Heistad, D. (1985) Regional, segmental and temporal heterogeneity of cerebral vascular autoregulation. Ann. Biomed. Eng. 13, 303-310.

Bayliss, W. (1902) On the local actions of the arterial wall to changes in internal pressure. J. Physiol. (Lond) 28, 220-231.

Borg, E. (1981) Physiological and pathogenic effects of sound. Acta Otolaryngol. Suppl. 381, 1-68.

Chang, B., Santillan, G. and Bing, R. (1985) Red cell velocity and autoregulation in the cerebral cortex of the cat. Brain Res. 308, 15-24.

Coyle, P. and Heistad, D. (1986) Blood flow through cerebral collateral vessels in hypertensive and normotensive rats. Hypertension Suppl. II, 8, 1167-1171.

Dengerink, H.A., Wright, J.W., Thompson, P. and Dengerink, J.E. (1982) Changes in plasma angiotensin II with noise exposure and their relationship to TTS. J. Acoust. Soc. Am. $72,276-278$.

Dengerink, H., Wright, J., Miller, J. and Goodwin, P. (1985) The effects of nicotine on laser Doppler measures of cochlear blood flow. Hear. Res. 20, 31-36.

Drettner, B., Hedstrand, H. and Klockhoff, I. (1975) Cardiovascular risk factors and hearing loss. A study of 1000 fifty year old men. Acta Otolaryngol. (Stockh.) 79, 366-371.

Goodwin, P., Miller, J., Dengerink, H., Wright, J. and Axelsson, A. (1984) The laser Doppler: a non-invasive measure of cochlear blood flow. Acta Otolaryngol. 98, 403-412.

Harper, S. and Bohlen, G. (1981) Microvascular adaptation in the cerebral cortex of adult spontaneously hypertensive rats. Hypertension 6, 408-419.

Harper, S., Bohlen, G. and Rubin, M. (1984) Arterial and microvascular contributions to cerebral cortical autoregulation in rats. Am. J. Physiol. 246, H17-H24.

Hultcrantz, E. and Nuttall, A.L. (1987) Effect of hemodilution of cochlear blood flow measured by laser-Doppler flowmetry. Am. J. Otolaryngol. 8, 10-16.

Johnsson, A. and Hansson, L. (1977) Prolonged exposure to a stressful stimulus (noise) as a cause of raised blood pressure in man. Lancet 8,86 .

Lawrence, M., Nuttall, A.L. and Burgio, P.A. (1977) Oxygen reserve and autoregulation in the cochlea. Acta Otolaryngol. 83, 146-152.

Miller, J.M., Marks, N.J. and Goodwin, P.C. (1983) Laser Doppler measurements of cochlear blood flow. Hear. Res. 11, 385-394.

Miller, J.M., Goodwin, P.C. and Marks, N.J. (1984) Inner ear blood flow measured with a laser Doppler system. Arch. Otolaryngol. 110, 305-308.

Nuttall, A.L. (1987) Velocity of red blood cell flow in capillaries of the guinea pig cochlea. Hear. Res. 27, 121-128.

Parvispoor, D. (1976) Noise exposure and prevalence of high blood pressure among weavers in Iran. J. Occup. Med. 18, $730-731$.

Pasternak, J.F. and Groothuis, D.R. (1985) Autoregulation of cerebral blood flow in the newborn beagle puppy. Biol. Neonate 48, 100-109.

Pillsbury, H.C. (1986) Hypertension, hyperlipoproteinemia, chronic noise exposure: is there synergism in cochlear pathology. Laryngoscope 96, 1112-1138.

Quirk, W.S., Bademian, M., Wright, J.W. and Dengerink, H.A. (1988) Effects of mannitol and dextran on cochlear blood 
flow in normotensive and spontaneously hypertensive rats. (submitted).

Sadoshima, S., Busija, D. and Heistad, D. (1985) Mechanisms of protection against stroke in stroke-prone spontaneously hypertensive rats. Am. J. Physiol. 244, H406-H412.

Shiffrin, E.L., Thome, F.S. and Genest, J. (1984) Vascular angiotensin II receptors in SHR. Hypertension 6, 682-688.

Strangaard, S., Jones, J.V., MacKenzie, E.T. and Harper, A.M. (1975) Upper limit of cerebral blood flow autoregulation in experimental renovascular hypertension in the baboon. Circ. Res. 46, 37-47.

Williams, H.L. (1965) A review of the literature as to the physiologic dysfunction of Meniere's disease: A new hypothesis as to its fundamental cause. Laryngoscope 75, 1661.

Wright, J.W., Dengerink, H.A., Thompson, P. and Morseth, S.
(1981) Plasma angiotensin II changes with noise exposure at three levels of ambient temperature. J. Acoust. Soc. Am. 70, 1353-1356.

Wright, J.W., Dengerink, H.A., Miller, J.M. and Goodwin, P.C. (1985a) Potential role of angiotensin II in noise-induced increases in inner ear blood flow. Hear. Res. 17, 41-46.

Wright, J.W., Morseth, S.L., Abhold, R.H. and Harding, J.W. (1985b) Pressor action and dipsogenicity induced by angiotensin II and III in rats. Am. J. Physiol. 249, R514-R521.

Yamori, Y. (1983) Physiopathology of various strains of spontaneously hypertensive rat. In: J. Genest, O. Kuchei, P. Hamlet and M. Cantin (Eds.), Hypertension Physiopathology and Treatment, McGraw-Hill, New York, pp. 556-581. 